$\epsilon$ Aurigæ is known to be a supergiant star. Its atmospheric density must consequently be very low. This seems to support the idea, expressed first by Unsöld, that the observed residual intensities in the lines of other stars are due to some process depending upon collisions between the atoms. The fact that the hydrogen line in $\epsilon$ Aurigæ is free from the wide wings which are characteristic for other stars and are apparently produced by intra-molecular Stark effect, is itself a good indication that the pressure is very low.

O. STRUVE.

C. T. Elvey.

Yerkes Observatory of the

University of Chicago, Jan. 7.

\section{Zygospore Formation in Mucors.}

SINCE the discovery of heterothallism, or separate sexes, in Mucors by Blakeslee some twenty-five years ago, the conception has been widely extended in the fungi and has recently been applied to the green algæ. But in recent years the facts regarding multiple sexes in fungi have become so complex as to strain credulity, and new views, such as Dame Helen Gwynne-Vaughan's conception of nutritive heterothallism, have been coming in to relieve the tension on the earlier rigid hypothesis of fixed + and - strains corresponding respectively to the female and the male sex.

Finding peculiar results from certain strains of Mucor hiemalis which we have had for some years in this laboratory, it was decided to make an analytical study of the process of zygospore formation in this species in relation to various media. Several other genera of Mucorineæ have also been investigated at the same time. Subcultures were made from the original stocks, and subsequent monospore cultures from these. Experiments were made, not only varying the environmental conditions such as temperature, humidity, and light, but also to show the effects of adding various substances to the agar culture medium. A few only of these experiments can be summarised here.

In the heterothallic Mucor hiemalis, zygospore formation is most favoured when the moisture content of the medium is at a minimum, and the humidity can be regulated by varying the percentage of agar used in making up the medium. The addition of zinc nitrate, on the contrary, appears to increase the development of mycelium and sporangia. Most of the species thrive best on an alkaline medium $(p \mathrm{H} 7 \cdot 2)$. When + and - strains of Mucor hiemalis are grown together in a Petrie dish on potato agar at a temperature of about $15^{\circ} \mathrm{C}$., zygospore formation appears to be completely inhibited; but identical cultures grown at $25^{\circ} \mathrm{C}$. produce large numbers of zygospores. At low temperature also the two strains will not intermingle, but a line of repulsion or aversion remains between them, which appears to be due to the fact that the medium has been rendered toxic. When such cultures are placed in an incubator at higher temperature they produce zygospores.

Small doses of oxalic, formic, malic, citric, or hydrochloric acids added to the medium so as to change the $p \mathrm{H}$ from 7.2 to 6.8 have a toxic effect on the fungus, causing repulsion in every case; that is, with + and + , or - and -, or + and -. How ever, like strains grown on a medium containing starch ( 2 per cent), agar ( 1.5 per cent), and diastase (0.04 per cent) will show no signs of repulsion, but no zygospores are formed except between + and strains. Different media produce striking effects on the morphological characters of the mycelium. Media having sugars or traces of acids produce enormous oil inclusions in the hyphæ, which are cylindrical, thick, and heavy in appearance, while on a medium without sugar or acid the hyphæ are flat, hyaline, and much less branched. In starch diastase medium the hyphæ become septate and the protoplasm highly granular.

It is found that the mycelia of + and - Mucor, Rhizopus, Sporodinia, and other Mucorineæ all produce two kinds of sporangia, namely, large and small, the large sporangia emitting large spores and the small sporangia small ones.

Perhaps the most striking result has been the production of imperfect zygospores from the - strain of $M$. hiemalis alone when grown on a medium composed of 1 per cent fructose, 1.2 per cent agar and a few drops of acetic acid bringing the $p \mathbf{H}$ to 6.8. These zygospores ceased development after fusion of the progametes had taken place, and the wall had begun to thicken. The process resembles the 'imperfect hybridisation' obtained by Blakeslee by bringing together + and - strains from different genera. In no case has zygospore formation been obtained between + strains. Chlamydospores are frequently produced in all the strains, and under certain conditions azygospores are formed.

By fixing the mycelium in 3-5 per cent formalin and staining in Ehrlich's hæmatoxylin, the nuclei in zygospore formation can be followed under an immersion lens. The pronuclei remain separate, although in close contact, so far as their history has yet been followed. Several zygospores have been germinated in hanging drop cultures by a method to be described later, and it is hoped to follow the later history of the nuclei.

This work is being extended in various directions and a full account will be published later.

R. Ruggles Gates.

King's College, London,

D. V. Daran. Jan. 31 .

The Viscosity of Liquids.

THe viscosity of liquids is a subject which, so far as I know, has hitherto been without any general theoretical basis. No physical mechanism, for example, has ever been brought forward to account even for so simple and general a fact as the decrease of liquid viscosity with temperature, in contrast to the familiar and well explained increase of gaseous viscosity with temperature. As a result, the study has hitherto consisted largely of a collection of more or less well established empirical relations, mostly of limited scope.

In consequence of certain general theoretical considerations, shortly to be published, which I have been applying to the problem of liquid viscosity, I have not only been able to give a general account of the influence of temperature on liquid viscosity, but also I have arrived at a formula of great simplicity which fits the experimental facts very well. There is, of course, a whole collection of empirical formulæ (due to Poiseuille, Koch, Meyer, Slotte, and others-see, for example, Hatschek's "The Viscosity of Liquids" (1928)) connecting viscosity with temperature, but they have all at least three arbitrary constants, and a very limited range of validity. Different types of formula have been employed for different liquids. My formula has only two constants, so that the agreement between it and the experiment emboldens me to believe that it has some fundamental significance, as indicated by my theory. The formula is

$$
\eta=A e^{b / T}
$$

where $\eta$ is the viscosity, $T$ the absolute temperature, and $A$ and $b$ the constants. It holds over practically 